\title{
CURVAS DE APALANCAMIENTO Y ELECCIÓN DE CARTERAS EN LA BMV
}

Jorge Ludlow Wiechers*

Departamento de Economía

Universidad Autónoma Metropolitana-Atzcapotzalco

M. Beatríz Mota Aragón

Departamento de Economía

Universidad Autónoma Metropolitana-Iztapalapa

(Recibido 27 de octubre 2005, aceptado 8 de diciembre 2005)

\section{Resumen}

En este trabajo estudiaremos el efecto apalancamiento conocido también como volatilidad asimétrica, el cual se presenta en los mercados de capital y se refiere a la correlación negativa entre los rendimientos pasados y la volatilidad futura de las acciones. Estimaremos la asimetría de la volatilidad con los modelos GARCH asimétrico de Glosten, Jagannathan y Runkle (1993) y el modelo GARCH exponencial (EGARCH) de Nelson (1991). Utilizamos los precios de operación diarios de las 196 acciones que cotizan en la Bolsa Mexicana de Valores, de los cuales seleccionamos una muestra de 62 por su bursatilidad. Finalmente proponemos usar las opciones put como estrategia de administración de riesgo bajo el efecto apalancamiento.

\section{Abstract}

In this work we study the leverage effects also known as asymmetry volatility. Which is present in capital markets and it is about the negative correlation between past returns and future stock volatility. Volatility asymmetries are estimated based upon the asymmetric GARCH model from Glosten, Jagannathan y Runkle (1993) and the exponential GARCH (EGARCH) from Nelson (1991). Data came from diary operation prices that conform the Mexican Stock Market, because the level of tradability we select a sample of 62 firms, from 196. Finally, we suggest to use put financial options as risk administrative strategies.

Clasificación JEL: C32, G11, G14

Palabras clave: Volatilidad asimétrica, Efecto apalancamiento, Modelo GARCH simétrico, Modelo GARCH exponencial

* Depto. Economía. Div. CSH UAM-Azc. Av. San Pablo no. 180. Col. Reynosa Tamaulipas. C.P. 02200, México D.F. Teléfono +52 (55)5318-9424. Correo electrónico: jlw@correo.azc.uam.mx y macroescenarios@yahoo.com 


\section{Introducción}

Las economías de mercado se caracterizan por un alto grado de imperfecciones. La tesis de esta escuela de pensamiento económico contemporánea consiste en dos fundamentales: Primero, la presencia de la asimetría en la información para los agentes económicos y segundo, la formación de los precios, el crecimiento, la distribución del ingreso, los ciclos y la política económica, Akerlof (1970), [Spence (1976)] y [Stiglitz (1985)].

El modelo de información asimétrica representa una ruptura radical con la teoría neoclásica de los precios ya que postula microfundamentos de competencia imperfecta al análisis económico, en donde otras funciones económicas determinan el resultado macroeconómico global. Con ello demuestra (contrario a la hipótesis fundamental de la teoría walrasiana del equilibrio general) que la economía de mercado tiende espontáneamente a trampas de equilibrio macroeconómico subóptimos, es decir, a un equilibrio derivado del intercambio y del mecanismo de precios que no es socialmente eficiente (la eficiencia en el sentido de Pareto), Perrotini.

La información asimétrica describe una forma de falla de mercado. Se trata de mercados imperfectos donde los participantes no tienen el mismo grado de información. La información se presenta en la forma de selección adversa y riesgo moral (Akerlof, 1970). El primer problema corresponde a la teoría de mercados con información asimétrica y el segundo a la teoría de la agencia. En presencia de información asimétrica los agentes económicos no pueden calcular los precios sombra y, consecuentemente, sus decisiones generan procesos de selección adversa, racionamiento y daño moral. Nos ocupa el primer problema, y en teoría financiera, una explicación a la asimetría de la información es el efecto apalancamiento, motivo del presente estudio.

El trabajo esta dividido en cuatro secciones más: La segunda nos orienta hacia el modelo teórico de la investigación. En la tercera sección describimos la metodología, utilizaremos los modelos GARCH asimétrico y EGARCH para estimar la asimetría de la volatilidad del IPC y de la muestra seleccionada. La cuarta analiza los resultados. Por último, presentamos las conclusiones.

\section{Hacia el modelo teórico}

Una cantidad importante de estudios se han dirigido a modelar el efecto asimétrico de noticias sobre la volatilidad de los rendimientos de las acciones. Estos se orientan básicamente al estudio de acciones individuales; Black (1976), Christie (1982), y Cheung y Ng (1992), y al estudio de índices accionarios; Frech, Schwert y Stambaugh (1987), Nelson (1991), Engle y Ng (1993), y Glosten, Jagannathan y Runkle (1993).

El efecto asimétrico sobre la volatilidad se dirige al estudio del apalancamiento financiero y al estudio de los determinantes de la prima de riesgo. El primer enfoque argumenta que el grado de apalancamiento de la estructura de capital de la empresa afecta el precio de la acción, y consecuentemente el nivel de la volatilidad, Black (1976) y Christie (1982). El segundo se dirige a la relación positiva entre la volatilidad y la prima de riesgo esperada. Poterba y Summers (1986), Bollerslev, Engle y Wooldridge (1988).

En el estudio de las acciones individuales, Black (1976) argumento que la volatilidad de las acciones tiende a incrementarse cuando los rendimientos 
son negativos y desciende cuando los rendimientos son positivos. Es importante notar que el efecto apalancamiento sólo explica parcialmente la fuerte correlación negativa entre los rendimientos presentes y la volatilidad presente de las acciones, Black (1976) y Christic (1982). En contraste con la relación causal negativa de los rendimientos pasados y la volatilidad futura.

En el estudio de índices accionarios Glosten, Jagannathan y Runkle (1993) observaron que la volatilidad se incrementa cuando el precio de las acciones disminuye (los rendimientos son negativos), entonces se incrementan los rendimientos requeridos sobre el capital, haciendo que la acción tenga mayor riesgo e incrementando el apalancamiento financiero. Tales relaciones podrían ser consistentes con el CAMP, [Sharpe (1964)], aunque controlan un equilibrio de mercado bajo supuestos restrictivos. Pindyck (1988) observó que la volatilidad pasada se relaciona positivamente con el exceso de rendimientos porque estos contienen información acerca de la varianza condicional. Esto es, una innovación positiva en la varianza hoy implica una volatilidad futura elevada y entonces, altos rendimientos futuros. Cuando los rendimientos futuros son altos, la innovación en la varianza puede estar asociada con una baja en el índice accionario.

[Gallant, Rossi, y Tauchen (1990)] sugieren que el efecto apalancamiento en el índice diario del NYSE no es estadísticamente significativo. Es evidente que tanto en el estudio de acciones individuales como en el análisis de índices accionarios existe asimetría, lo que cambia es el nivel de análisis.

Poner atención en la asimetría de la volatilidad es importante porque ayuda a comprender el dinamismo del mercado, en este sentido apoya a la administración financiera a tomar mejores decisiones.

Es claro que la asimetría de la volatilidad está relacionada con los efectos de la información sobre el precio de la acción y su volatilidad. Los choques positivos se refieren a las buenas noticias y los choques negativos a las malas noticias, la presencia de cualquiera de los dos incrementa la volatilidad actual y futura. La volatilidad es mayor cuando ocurre un choque negativo que cuando ocurre uno positivo. Para French, Schwert y Stambaugh (1987), los choques causados por la información simplemente reflejan la existencia de un cambio parcial en la prima de riesgo.

Una importante tarea para los administradores de riesgo ligados a la BMV, es tomar muy en cuenta la reacción de los mercados, ya que al ser correcta la afirmación: "las malas noticias repercuten en la volatilidad con mayor fuerza que las buenas noticias" la administración financiera podría salir equivocada.

Por último, una cualidad interesante de la volatilidad del mercado accionario es la persistencia de los choques de la varianza, Proterba y Summers (1986) argumentaron como los choques de las acciones tienden a persistir por un largo período de tiempo.

Ahora bien, la asimetría de la varianza se mide en las curvas de apalancamiento, Engle y $\mathrm{Ng}$ (1993) presentaron la curva del impacto de las noticias sobre la volatilidad con base en la cual proponen pruebas de diagnostico para observar la asimetría de la volatilidad. Las pruebas consisten en probar si los cuadrados de los residuales normalizados pueden ser predecibles con base en las variables históricas observadas no incluidas en el modelo. Las pruebas son las de sesgo en el signo, tanto negativo como positivo y se encargan de analizar los 
efectos de los choques (positivos y/o negativos) sobre la volatilidad y no están establecidos en el modelo a evaluar. En el trabajo se demuestra que los modelos GARCH asimétrico de Glosten, Jagannathan y Runkle (1993), así como el EGARCH de Nelson (1991), capturan exitosamente la asimetría.

\section{Metodología}

El uso de los modelos ARCH ha sido significativo para los resultados obtenidos en la teoría del precio de los activos, entre las teorías más prominentes que han encontrado implementaciones empíricas utilizando ARCH, tenemos: El CAMP; [Sharpe (1964)], [Lintner (1965)], el APT; [Ross (1976a), (1976b)], Black (1973), el modelo intertemporal (ICAMP); Merton (1973), el CAMP orientado al consumo; [Breeden (1979)].

Retomando a Merton (1973) quien construyó sobre el modelo intertemporal (ICAMP) para ilustrar la relación entre los rendimientos del mercado accionario y la volatilidad, y uso el modelo ARCH-M como una implementación del CAMP para mostrar que los inversionistas adversos al riesgo demandan una compensación extraordinaria igual al riesgo adicional; Merton (1980) argumentó una relación lineal positiva entre los rendimientos esperados y la varianza de la cartera del mercado y provee las condiciones bajo las cuales el componente de cobertura de los rendimientos extraordinarios es pequeño.

Engle, Lilien y Robins (1987) desarrollaron el modelo ARCH-M para estimar esta relación lineal utilizando el parámetro de aversión al riesgo para medir el impacto de la varianza sobre los rendimientos y encontraron una relación positiva. Sin embargo, otras investigaciones han argumentado una relación negativa entre los rendimientos y la varianza, Campbell (1987), Guo (2002) y Ng (1991).

No obstante los avances obtenidos, estos modelos presentan algunas limitaciones. El modelo GARCH $(\mathrm{p}, \mathrm{q})$ ha sido utilizado en otros estudios, [Kupiec (1990)]. Sin embargo, no captura totalmente la dinámica asimétrica porque la varianza condicional esta ligada únicamente a las varianzas condicionales pasadas y a las innovaciones cuadradas, por lo que el signo de los rendimientos no juega un papel importante en las volatilidades. Esta limitante de los modelos ARCH estándar es una de las motivaciones principales para el desarrollo de otras propuestas de extensión de los modelos GARCH; el GARCH de umbral (TARCH) de Glosten, Jagannathan y Runkle (1993) quienes argumentaron que la relación entre la volatilidad y los rendimientos esperados puede ser negativa, al igual que Nelson (1989) y de Zakoian (1990) y el modelo EGARCH de Nelson (1991). Como sabemos en este tipo de modelos ARCH, la volatilidad depende de la magnitud en los rendimientos pasados y de sus signos correspondientes.

En ambos modelos, como es usual, se hace la descomposición:

$$
\begin{gathered}
r_{t}=\mathrm{E}\left[r_{t} \mid \psi_{t-1}\right]+\varepsilon_{t} \\
\operatorname{Var}\left[\varepsilon_{t} \mid \psi_{t-1}\right]=h_{t}
\end{gathered}
$$

La primera relación nos dice que el rendimiento $\left(r_{t}=100 *\left\{\log \left(P_{t}\right)-\right.\right.$ $\left.\log \left(P_{t-1}\right)\right\}, P(t)$ es el precio de la acción al día $t$ y tiene una componente esperada, dada la toda información disponible $\psi_{t-1}$ hasta el tiempo $t-1$ y una innovación $\varepsilon_{t}=\left(\varepsilon_{i t}\right) i=1, \cdots, n$, que no es observable directamente. Por los 
segundos momentos está la varianza condicional, denotada por $h_{t}$, se obtiene ya que se utiliza toda la información disponible $\psi_{t-1}$ hasta el tiempo $t-1$. La información disponible incluye las buenas y las malas noticias.

En cifras diarias es conveniente tomar $\mathrm{E}\left[r_{t} \mid \psi_{t-1}\right]=\mu$ es el rendimiento esperado así la componente que tiene especial interés es $h_{t}$.

$$
\begin{gathered}
r_{t}=\mu+\varepsilon_{t} \\
\operatorname{Var}\left[\varepsilon_{t} \mid \psi_{t-1}\right]=h_{t}
\end{gathered}
$$

La innovación $\varepsilon_{t}$, es la parte ligada a la varianza condicional, $h_{t}$, depende del tiempo ya que al arribar nueva información las expectativas se modifican.

Estimaremos la asimetría de la volatilidad del modelo GARCH como:

$$
h_{t}=\omega+\alpha \varepsilon_{t-1}^{2}+\beta h_{t-1}+\gamma I\left(\varepsilon_{t-1}<0\right)
$$

Y la ecuación de noticias es:

$$
\begin{gathered}
h_{t}=A+\alpha \varepsilon_{t-1}^{2} \text { para } \varepsilon_{t-1}>0 \\
h_{t}=A+(\alpha+\gamma) \varepsilon_{t-1}^{2}<0 \text { para } \varepsilon_{t-1}<0
\end{gathered}
$$

donde

$$
A=\omega+\beta \sigma^{2}
$$

En el modelo exponencial, introducido por Nelson (1991), $h_{t}$ es una función asimétrica del pasado $\varepsilon_{t-1}$ y esta definido como:

$$
\log \left(h_{t}\right)=\omega+\alpha\left[\frac{\left|\varepsilon_{t-1}\right|}{\sqrt{h_{t-1}}}-\sqrt{2 / \pi}\right]+\beta \log \left(h_{t-1}\right)+\gamma\left(\frac{\varepsilon_{t-1}}{\sqrt{h_{t-1}}}\right)
$$

Y la ecuación de noticias es:

$$
\begin{aligned}
& h_{t}=A^{*} \exp \left[\frac{\alpha+\gamma}{\sigma} \varepsilon_{t-1}\right] \operatorname{para} \varepsilon_{t-1}>0 \\
& h_{t}=A^{*} \exp \left[\frac{\alpha-\gamma}{\sigma} \varepsilon_{t-1}\right] \operatorname{para} \varepsilon_{t-1}<0
\end{aligned}
$$

donde

$$
A=\sigma^{2 \beta} \exp (\varpi-\alpha \sqrt{2 / \pi})
$$

Podríamos llamar plus $=\alpha+\gamma$ y minus $=\alpha-\gamma$. La componente plus muestra el impacto a que es forzada la volatilidad bajo un choque positivo, mientras que la columna minus muestra el impacto de las malas noticias.

Note con cuidado el parámetro $\gamma$ en el primer caso altera la convexidad de la curva de noticias, mientras que en el modelo exponencial mide el impacto de los residuos estandarizados en la nueva volatilidad. En ambos casos cuando $\gamma=0$ sea nula, significa que no hay asimetría por lo que las buenas y malas 
noticias impactan por igual en la curva de noticias. Por esto será importante incluir la prueba de hipótesis $H_{0}: \gamma=0$

\section{Análisis del índice del mercado y de las emisoras}

Se revisaron las 196 acciones que cotizan en la BMV, las cuales presentamos enseguida:

\begin{tabular}{lll}
\hline ACCELSA B & COMERCI UB & GMACMA B \\
ACEYAC & COMERCI UBC & GMARTI \\
AGRIEXP A & CONTAL & GMD B \\
AHMSA & CONVER A & GMEXICO B \\
ALFA & COVARRA & GMODELO C \\
ALMACO 1 & CYDSASA A & GMODERN \\
ALMACO 2 & CYDSASA B & GNP \\
ALSEA & DERMET B & GOMO \\
AMTEL A1 & DESC A & GPH 1 \\
AMX A & DESC B & GPROFIN O \\
AMX L & DIANA A & GPROFUT \\
ARA & DIANA B & GRUMA B \\
ARCA & DIXON & GSANBOR B1 \\
ARISTOS A & ECE & HERDEZ \\
ARISTOS B & EDOARDO B & HILASAL A \\
ASUR B & EKCO & HOGAR B \\
AUTLAN B & ELEKTRA & HOMEX \\
BACHOCO UBL & EMPAQ B & HYLSAMX B \\
BAFAR B & FECHABASE & HYLSAMX L \\
BBVA & FEMSA UB & IASASA \\
BEVIDES A & FEMSA UBD & ICA \\
BEVIDES B & FIASA 1 & ICH B \\
BIMBO A & FIASA 2 & IEM A \\
BQ L & FOTOLUZ B & IEM B \\
BQ O & FRAGUA B & IMSA UBC \\
BUFETE CPO & GACCION B & INVEX O \\
C & GAM B & IPC \\
CABLE CPO & GCARSO A1 & IXEGF O \\
CAMPUS A & GCC & KIMBER A \\
CAMPUS B & GCORVI UBL & KIMBER B \\
CBARKA O & GEASA 1 & KOF L \\
CBESTRA A & GEASA 2 & LAMOSA B \\
CBESTRA A1 & GENSEG LASEG & TELMEX AA \\
\hline & & \\
\hline
\end{tabular}




\begin{tabular}{lll}
\hline CEL & MADISA B & SARE B \\
CEMEX A & MADISA L & SAVIA A \\
CEMEX B & MASECA A & SIMEC B \\
CEMEX CPO & MASECA B & SORIANA B \\
CERAMIC B & MEDICA B & SURESTE A \\
CERAMIC D & MEXCHEM & SURESTE B \\
CIDMEGA & MINSA B & SYNCRO A \\
CIE B & MINSA C & SYNCRO C \\
CINTRA A & MOVILA B & TEKCHEM A \\
CMOCTEZ & NAFTRAC 02 & TELECOM A1 \\
CMR B & NUTRISA & TELMEX A \\
CNCI B & PARRAS & TELMEX AA \\
CODUSA A & PATRIA & TELMEX L \\
CODUSA B & PENOLES & TLEVISA A \\
COLLADO & PERKINS A & TLEVISA CPO \\
GEO B & PERKINS B & TLEVISA D \\
GEUPEC B & PLAVICO & TLEVISA L \\
GFCREMI C & POSADAS A & TMM A \\
GFGBM L & POSADAS L & TRIBASA \\
GFGBM O & PROCORP A & TS \\
GFINBUR O & PROCORP B & TUACERO A \\
GFINTER O & PYP B & TUACERO B \\
GFMEXI C & Q CPO & TVAZTCA CPO \\
GFMEXI O & QBINDUS A & UNEFON A \\
GFMULT O & QBINDUS B & URBI \\
GFNORTE O & QTEL B & USCOM B1 \\
GFSCTIA B & QUMMA B & VALLE V \\
GICONSA & RCENTRO A & VALUEGF O \\
GIGANTE & REALTUR A & VIDEO \\
GISSA & SAB & VITRO A \\
LIVEPOL 1 & SANLUIS A & WALMEX V \\
LIVEPOL C1 & SANLUIS CPO & \\
LOTONAL & SANMEX B & \\
\hline
\end{tabular}

Lo primero que observamos en este contexto es que muchas acciones permanecen sin alterar su precio por largos periodos de tiempo, incluso meses, lo cual indica que no estuvieron a la venta. Para el análisis de volatilidad éstas tuvieron que descartarse, por lo que el universo de los títulos se redujo, construimos una nueva muestra con las acciones cuyo precio se mueve con frecuencia. En la práctica son las acciones que forman los fondos de inversión de renta variable.

Una componente obligada fue observar el tiempo de operación de la acción en el mercado. Por ejemplo, BBVA es de reciente incorporación, por lo tanto, los precios de sus cotizaciones tienen una historia muy corta y tuvo que descartarse. De tal manera solo analizamos series con 300 días operados o más. 
Otra actividad fue la de ajustar los días, un titulo puede no tener actividad un día, por lo tanto su precio permanece constante. Así los días con huecos (sin actividad) se consideraron con la cotización más reciente del mercado (último hecho).

La actividad real del mercado de capital (acciones con bursatilidad y con historia en el mercado) se concentra solamente en 62 emisoras de las 196 listadas, las cuales mencionamos en el cuadro 2:

\begin{tabular}{llll}
\hline ALFA & GSANBOR B1 & COMERCI UBC & SANLUIS CPO \\
AMTEL A1 & DESC B & HOGAR B & SIMEC B \\
AMX A & ELEKTRA & HOMEX & SORIANA \\
AMX L & FEMSA UBD & HYLSAMX B & TELECOM A1 \\
ARA & GCARSO A1 & HYLSAMX L & TELMEX A \\
ARCA & GCC & ICA & TELMEX L \\
ASUR B & GCORVI UBL & ICH B & TLEVISA CPO \\
BACHOCO UBL & GEO B & IMSA UBC & TS \\
BIMBO A & GFINBUR O & KIMBER A & TVAZTECA CPO \\
C & GFNORTE O & KOF L & URBI \\
CEL & GIGANTE & MASECA B & USCOM B1 \\
CEMEX CPO & GISSA & MOVILA B & VALLE V \\
CIE B & GMEXICO B & NAFTRAC O2 & VITRO A \\
CNCI B & GMODELO C & PENOLES & WALMEX V \\
COMERCI UBC & GRUMA B & SAN LUIS CPO & \\
CONTAL & GSANBOR B1 & SARE B & \\
\hline
\end{tabular}

Fuente: Elaboración propia

En esta muestra recae la operación del mercado en México, estas acciones son las que claramente compiten, o bien, son las que contrarestan la falta de competitividad de las 134 restantes. En este sentido vale la pena mencionar que si la reforma financiera hubiera continuado tal como se sugirió en el gobierno Zedillista el mercado bursátil estaría mucho más abierto y tendríamos la posibilidad de encontrar un mercado verdaderamente competido. Sin embargo, la lentitud con la que se ha venido dando este proceso en años posteriores limita la posibilidad de tener un mercado de tales magnitudes.

Ahora vamos a presentar los cuadros 3 y 4 con los resuitados de los modelos Garch asimétrico y Garch Exponencial para las emisoras más bursátiles de la Bolsa de Valores. El cuadro 3 contiene los resultados con el modelo Garch asimétrico; en este cuadro tiene especial atención el parámetro gama y su $t$ gama. Cuando el valor de $t$ es grande (digamos mayor a dos) es significativo el valor de gama, por lo que la curva de noticias si tiene un efecto asimétrico. Otro aspecto importante es comparar el valor de la beta que indica el Capital Asset Pricing Model (CAPM); note por ejemplo que la acción CEL es (muy) defensiva y al mismo tiempo gama no es significativa por lo que la curva de noticias de la acción CEL es simétrica. 
Cuadro 3. Los resultados del modelo Garch asimétrico para las 62 emisoras.

\begin{tabular}{lccccc}
\hline Emisora & media & omega & alfa & beta & gama \\
ALFA & 0.100 & 0.053 & 0.030 & 0.943 & 0.044 \\
AMTEL A & 10.206 & 0.304 & 0.039 & 0.800 & 0.159 \\
AMX A & -0.004 & 29.417 & 0.064 & -0.985 & 0.064 \\
AMX L & 0.116 & 0.088 & 0.058 & 1.005 & 0.055 \\
ARA & 0.068 & -0.004 & 0.000 & 1.001 & 0.001 \\
ARCA & 0.022 & 0.109 & 0.032 & 0.860 & 0.120 \\
ASUR B & 0.039 & 0.197 & 0.034 & 0.976 & 0.035 \\
BACHOCO UBL & 0.011 & 0.228 & 0.090 & 0.868 & 0.029 \\
BIMBO A & 0.032 & 0.143 & 0.050 & 0.891 & 0.061 \\
C & -0.042 & 0.011 & 0.021 & 0.928 & 0.104 \\
CEL & 1.825 & 869.619 & 0.094 & -0.611 & 0.042 \\
CEMEX CPO & 0.035 & 2.286 & 1.691 & 0.185 & 1.410 \\
CIE B & 0.035 & 0.091 & 0.018 & 0.919 & 0.104 \\
COMERCI UBC & 0.035 & 0.097 & 0.062 & 0.893 & 0.062 \\
CONTAL & -0.044 & 13.391 & 0.049 & -0.741 & 0.047 \\
CYDSASA A & -0.190 & 10.223 & 0.346 & 0.261 & 0.353 \\
DESC B & -0.130 & 43.038 & 0.049 & -1.001 & 0.049 \\
ELEKTRA & 0.078 & 6.910 & 1.014 & 0.000 & 0.772 \\
FEMSA UBD & 0.114 & 4.012 & 15.346 & 0.001 & 15.042 \\
GCARSO A1 & -0.017 & 6.681 & 0.064 & 0.474 & 0.063 \\
GCC & 0.103 & 0.060 & 0.173 & 0.828 & 0.019 \\
GCORVI UBL & -0.003 & 4.896 & 0.244 & 0.006 & 0.046 \\
GEO B & 0.154 & 1.855 & 0.261 & 0.194 & 5.767 \\
GFINBUR O & -0.025 & 7.703 & 0.069 & 0.305 & 0.068 \\
GFNORTE O & 0.139 & 0.056 & 0.008 & 0.950 & 0.069 \\
GIGANTE & 0.044 & 14.416 & 0.028 & -0.034 & 0.032 \\
GISSA & -0.067 & 0.573 & 0.304 & 0.402 & 0.065 \\
GMEXICO B & 0.189 & 0.167 & 0.097 & 1.008 & 0.094 \\
GMODELO C & 0.003 & 18.573 & 0.036 & -1.000 & 0.035 \\
GRUMA B & 0.001 & 0.086 & 0.073 & 0.902 & 0.017 \\
GSANBOR B1 & 0.015 & 0.024 & 0.031 & 0.940 & 0.058 \\
HILASAL A & -0.105 & 0.186 & 0.111 & 0.879 & 0.013 \\
HOGAR B & -0.093 & 3.379 & 0.182 & 0.514 & 0.056 \\
\hline & & & & & \\
\hline
\end{tabular}


Cuadro 3. (continuación).

\begin{tabular}{lcccc}
\hline Emisora & t-gama & $\sigma^{2}$ & betacapm & CoefDet \\
ALFA & 5.465 & 9.233 & 1.059 & 0.343 \\
AMTEL A & 4.852 & 3.737 & 1.422 & 0.569 \\
AMX A & 13.847 & 3.602 & 1.065 & 0.099 \\
AMX L & 63.002 & 4.227 & 1.439 & 0.188 \\
ARA & 1.319 & 6.070 & 0.684 & 0.106 \\
ARCA & 4.899 & 3.024 & 0.333 & 0.054 \\
ASUR B & 25.023 & 8.955 & 0.211 & 0.009 \\
BACHOCO UBL & 3.408 & 10.053 & 0.253 & 0.040 \\
BIMBO A & 4.758 & 5.397 & 0.620 & 0.211 \\
C & 6.391 & 3.339 & 0.800 & 0.223 \\
CEL & 0.249 & 1965.723 & -0.288 & 0.000 \\
CEMEX CPO & 14.379 & 4.969 & 0.944 & 0.325 \\
CIE B & 10.105 & 7.857 & 1.084 & 0.414 \\
CNCI B & 2.266 & 21.517 & 0.940 & 0.089 \\
COMERCI UBC & 5.081 & 6.531 & 0.758 & 0.271 \\
CONTAL & 4.760 & 4.713 & 0.467 & 0.074 \\
CYDSASA A & 9.017 & 10.137 & 0.280 & 0.012 \\
DESC B & 13.334 & 6.878 & 0.811 & 0.077 \\
ELEKTRA & 12.641 & 51.863 & 1.145 & 0.097 \\
FEMSA UBD & 15.608 & 5.787 & 1.019 & 0.080 \\
GCARSO A1 & 3.716 & 5.172 & 1.004 & 0.211 \\
GCC & 0.970 & 3.382 & 0.287 & 0.053 \\
GCORVI UBL & 0.978 & 3.774 & 0.388 & 0.062 \\
GEO B & 21.028 & 6.613 & 0.855 & 0.043 \\
GFINBUR & 6.164 & 3.442 & 0.619 & 0.073 \\
GFNORTE O & 7.910 & 6.868 & 0.985 & 0.331 \\
GIGANTE & 1.709 & 53.200 & 0.282 & 0.015 \\
GISSA & 1.147 & 0.996 & 0.232 & 0.028 \\
GMEXICO B & 28.070 & 5.091 & 0.914 & 0.037 \\
GMODELO C & 68.814 & 4.250 & 0.421 & 0.034 \\
GRUMA B & 2.080 & 6.166 & 0.358 & 0.082 \\
GSANBOR B & 8.743 & 5.925 & 0.624 & 0.177 \\
HILASAL A & 1.613 & 7.188 & 0.541 & 0.078 \\
HOGAR B & 1.740 & 9.644 & 0.500 & 0.055 \\
\hline & & & & \\
\hline
\end{tabular}


Cuadro 3. (continuación).

\begin{tabular}{lccccc}
\hline Emisora & media & omega & alfa & beta & gama \\
HOMEX & 0.160 & 0.798 & 0.335 & 0.562 & 0.085 \\
HYLSAMX B & 0.136 & 0.246 & 0.107 & 0.851 & 0.091 \\
HYLSAMX L & 0.009 & 0.000 & 0.423 & 0.651 & 0.995 \\
ICA & -0.097 & 3.218 & 0.190 & 0.566 & 0.020 \\
ICH B & -0.020 & 7.389 & 0.013 & 0.338 & 0.017 \\
IMSA UBC & 0.029 & 0.202 & 0.039 & 0.896 & 0.044 \\
KIMBER A & 0.010 & 0.045 & 0.031 & 0.923 & 0.067 \\
KOF L & 0.044 & 0.004 & 0.011 & 0.988 & 0.000 \\
MASECA B & 0.066 & 0.028 & 0.045 & 0.942 & 0.023 \\
MOVILA B & -0.170 & 6.155 & 12.552 & 0.066 & 12.188 \\
NAFTRAC 02 & 0.088 & 0.093 & 0.056 & 0.851 & 0.223 \\
PENOLES & -0.027 & 4.172 & 0.153 & 0.317 & 0.024 \\
SANLUIS CPO & -0.100 & 6.171 & 0.067 & 0.506 & 0.076 \\
SARE B & 0.050 & 1.119 & 0.131 & 0.425 & 0.046 \\
SAVIA A & 0.000 & 0.010 & 4.963 & 0.514 & 2.702 \\
SIMEC B & 0.564 & 0.190 & 0.000 & 1.002 & 0.023 \\
SORIANA B & 0.039 & 0.065 & 0.017 & 0.934 & 0.067 \\
TELECOM A1 & -0.113 & 8.061 & 1.673 & -0.008 & 1.672 \\
TELMEX A & -0.026 & 10.362 & 0.070 & 0.074 & 0.107 \\
TELMEX L & -0.044 & 9.102 & 0.055 & 0.131 & 0.053 \\
TLEVISA CPO & 0.072 & 0.013 & 0.018 & 0.966 & 0.028 \\
TS & 0.280 & 3.751 & 0.017 & 0.113 & 0.035 \\
TVAZTCA CPO & 0.037 & 0.160 & 0.065 & 0.902 & 0.045 \\
URBI & 0.209 & 0.536 & 0.061 & 0.734 & 0.011 \\
USCOM B1 & 0.023 & 1.538 & 0.247 & 0.133 & 0.327 \\
VALLE V & -0.050 & 1.509 & 0.309 & 0.619 & 0.124 \\
VITRO A & -0.050 & 0.196 & 0.083 & 0.894 & 0.004 \\
WALMEX V & 0.060 & 0.035 & 0.038 & 0.931 & 0.042 \\
\hline
\end{tabular}


Cuadro 3. (continuación).

\begin{tabular}{lcccc}
\hline Emisora & $t$-gama & $\sigma^{2}$ & betacapm & CoefDet \\
HOMEX & 0.991 & 5.510 & 0.721 & 0.106 \\
HYLSAMX B & 5.280 & 15.474 & 0.692 & 0.058 \\
HYLSAMX L & 5.959 & 5.011 & 0.903 & 0.136 \\
ICA & 0.653 & 12.805 & 0.886 & 0.157 \\
ICH B & 0.672 & 3.520 & 0.499 & 0.058 \\
IMSA UBC & 5.967 & 6.734 & 0.376 & 0.072 \\
KIMBER A & 7.291 & 4.099 & 0.703 & 0.315 \\
KOF L & 0.175 & 7.676 & 0.526 & 0.127 \\
MASECA B & 4.059 & 9.858 & 0.397 & 0.063 \\
MOVILA B & 34.977 & 46.312 & 0.397 & 0.008 \\
NAFTRAC 02 & 6.130 & 1.005 & 0.937 & 0.913 \\
PENOLES & 0.850 & 6.985 & 0.354 & 0.039 \\
SANLUIS CPO & 3.342 & 8.821 & 0.266 & 0.012 \\
SARE B & 0.588 & 3.893 & 0.699 & 0.172 \\
SAVIA A & 54.466 & 27.788 & 0.405 & 0.009 \\
SIMEC B & 65.419 & 288.922 & 0.462 & 0.008 \\
SORIANA B & 6.130 & 5.477 & 0.913 & 0.420 \\
TELECOM A1 & 14.226 & 5.898 & 1.074 & 0.179 \\
TELMEX A & 4.629 & 3.443 & 0.718 & 0.122 \\
TELMEX L & 6.033 & 4.132 & 0.992 & 0.249 \\
TLEVISA CPO & 6.366 & 7.951 & 1.269 & 0.551 \\
TS & 1.513 & 11.097 & 0.240 & 0.012 \\
TVAZTCA CPO & 4.688 & 11.617 & 1.254 & 0.397 \\
URBI & 0.262 & 3.374 & 0.404 & 0.055 \\
USCOM B1 & 2.983 & 5.785 & 0.385 & 0.056 \\
VALLE V & 6.140 & 5.921 & 0.146 & 0.006 \\
VITRO A & 0.377 & 7.442 & 0.562 & 0.109 \\
WALMEX V & 3.055 & 3.821 & 1.092 & 0.524 \\
\hline
\end{tabular}

De esta tabla seleccionamos solo las que el parámetro de asimetría es significativo, la emisora es eliminada si se acepta la hipótesis nula $H_{0}: \gamma=0$. Ahora para presentar la tabla, la ordenamos respecto a la columna beta-capm, esta es la beta que se obtiene del modelo auxiliar "market model".

$$
r_{t}=\beta r_{M_{t}}+\varepsilon_{t}
$$

La tabla presenta solo las 45 emisoras con un efecto asimétrico significante y ordenadas de agresivas a defensivas. Con esto para armar una cartera el inversionista tiene una mejor información. 
Cuadro 3a. Efecto asimétrico para empresas ordenadas de agresivas a defensivas.

\begin{tabular}{lccccc}
\hline Emisora & media & omega & alfa & beta & gama \\
AMX L & 0.12 & 0.09 & 0.06 & 1.00 & 0.06 \\
AMTEL A1 & 0.21 & 0.30 & 0.04 & 0.80 & 0.16 \\
TLEVISA CPO & 0.07 & 0.01 & 0.02 & 0.97 & 0.03 \\
TVAZTCA CPO & 0.04 & 0.16 & 0.06 & 0.90 & 0.04 \\
ELEKTRA & 0.08 & 6.91 & 1.01 & 0.00 & 0.77 \\
WALMEX V & 0.06 & 0.04 & 0.04 & 0.93 & 0.04 \\
CIE B & 0.04 & 0.09 & 0.02 & 0.92 & 0.10 \\
TELECOM A1 & -0.11 & 8.06 & 1.67 & -0.01 & 1.67 \\
AMX A & 0.00 & 29.42 & 0.06 & -0.99 & 0.06 \\
ALFA & 0.10 & 0.05 & 0.03 & 0.94 & 0.04 \\
FEMSA UBD & 0.11 & 4.01 & 15.35 & 0.00 & 15.04 \\
GCARSO A1 & -0.02 & 6.68 & 0.06 & 0.47 & 0.06 \\
TELMEX L & -0.04 & 9.10 & 0.06 & 0.13 & 0.05 \\
GFNORTE O & 0.14 & 0.06 & 0.01 & 0.95 & 0.07 \\
CEMEX CPO & 0.04 & 2.29 & 1.69 & 0.18 & 1.41 \\
CNCI B & -0.28 & 1.67 & 0.31 & 0.69 & 0.05 \\
NAFTRAC 02 & 0.09 & 0.09 & 0.06 & 0.85 & 0.22 \\
GMEXICO B & 0.19 & 0.17 & 0.10 & 1.01 & 0.09 \\
SORIANA B & 0.04 & 0.06 & 0.02 & 0.93 & 0.07 \\
HYLSAMX L & 0.01 & 0.00 & 0.42 & 0.65 & 0.99 \\
GEO B & 0.15 & 1.85 & 0.26 & 0.19 & 5.77 \\
DESC B & -0.13 & 43.04 & 0.05 & -1.00 & 0.05 \\
C & -0.04 & 0.01 & 0.02 & 0.93 & 0.10 \\
COMERCI UBC & 0.03 & 0.10 & 0.06 & 0.89 & 0.06 \\
TELMEX A & -0.03 & 10.36 & 0.07 & 0.07 & 0.11 \\
KIMBER A & 0.01 & 0.04 & 0.03 & 0.92 & 0.07 \\
HYLSAMX B & 0.14 & 0.25 & 0.11 & 0.85 & 0.09 \\
GSANBOR B1 & 0.02 & 0.02 & 0.03 & 0.94 & 0.06 \\
BIMBO A & 0.03 & 0.14 & 0.05 & 0.89 & 0.06 \\
GFINBUR O & -0.03 & 7.70 & 0.07 & 0.30 & 0.07 \\
CONTAL & -0.04 & 13.39 & 0.05 & -0.74 & 0.05 \\
SIMEC B & 0.56 & 0.19 & 0.00 & 1.00 & 0.02 \\
GMODELO C & 0.00 & 18.57 & 0.04 & -1.00 & 0.04 \\
SAVIA A & 0.00 & 0.01 & 4.96 & 0.51 & 2.70 \\
\hline & & & & & \\
\hline
\end{tabular}


Cuadro 3a. (continuación).

\begin{tabular}{|c|c|c|c|c|}
\hline Emisora & t-gama & $\sigma^{2}$ & betacapm & CoefDet \\
\hline AMX L & 63.00 & 4.23 & 1.44 & 0.19 \\
\hline AMTEL A1 & 4.85 & 3.74 & 1.42 & 0.57 \\
\hline TLEVISA CPO & 6.37 & 7.95 & 1.27 & 0.55 \\
\hline TVAZTCA CPO & 4.69 & 11.62 & 1.25 & 0.40 \\
\hline ELEKTRA & 12.64 & 51.86 & 1.14 & 0.10 \\
\hline WALMEX V & 3.06 & 3.82 & 1.09 & 0.52 \\
\hline CIE B & 10.10 & 7.86 & 1.08 & 0.41 \\
\hline TELECOM A1 & 14.23 & 5.90 & 1.07 & 0.18 \\
\hline AMX A & 13.85 & 3.60 & 1.06 & 0.10 \\
\hline ALFA & 5.46 & 9.23 & 1.06 & 0.34 \\
\hline FEMSA UBD & 15.61 & 5.79 & 1.02 & 0.08 \\
\hline GCARSO A1 & 3.72 & 5.17 & 1.00 & 0.21 \\
\hline TELMEX L & 6.03 & 4.13 & 0.99 & 0.25 \\
\hline GFNORTE O & 7.91 & 6.87 & 0.99 & 0.33 \\
\hline CEMEX CPO & 14.38 & 4.97 & 0.94 & 0.32 \\
\hline CNCI B & 2.27 & 21.52 & 0.94 & 0.09 \\
\hline NAFTRAC 02 & 613 & 1.00 & 0.94 & 0.91 \\
\hline GMEXICO B & 28.07 & 5.09 & 0.91 & 0.04 \\
\hline SORIANA B & 6.13 & 5.48 & 0.91 & 0.42 \\
\hline HYLSAMX L & 5.96 & 5.01 & 0.90 & 0.14 \\
\hline GEO B & 21.03 & 6.61 & 0.86 & 0.04 \\
\hline DESC B & 13.33 & 6.88 & 0.81 & 0.08 \\
\hline $\mathrm{C}$ & 6.39 & 3.34 & 0.80 & 0.22 \\
\hline COMERCI UBC & 5.08 & 6.53 & 0.76 & 0.27 \\
\hline TELMEX A & 4.63 & 3.44 & 0.72 & 0.12 \\
\hline KIMBER A & 7.29 & 4.10 & 0.70 & 0.31 \\
\hline HYLSAMX B & 5.28 & 15.47 & 0.69 & 0.06 \\
\hline GSANBOR B1 & 8.74 & 5.93 & 0.62 & 0.18 \\
\hline BIMBO A & 4.76 & 5.40 & 0.62 & 0.21 \\
\hline GFINBUR O & 6.16 & 3.44 & 0.62 & 0.07 \\
\hline CONTAL & 4.76 & 4.71 & 0.47 & 0.07 \\
\hline SIMEC B & 65.42 & 288.92 & 0.46 & 0.01 \\
\hline GMODELO C & 68.81 & 4.25 & 0.42 & 0.03 \\
\hline SAVIA A & 54.47 & 27.79 & 0.40 & 0.01 \\
\hline
\end{tabular}


Cuadro 3a. (continuación).

\begin{tabular}{lccccc}
\hline Emisora & media & omega & alfa & beta & gama \\
MASECA B & 0.07 & 0.03 & 0.04 & 0.94 & 0.02 \\
MOVILA B & -0.17 & 6.15 & 12.55 & 0.07 & 12.19 \\
USCOM B1 & 0.02 & 1.54 & 0.25 & 0.13 & 0.33 \\
IMSA UBC & 0.03 & 0.20 & 0.04 & 0.90 & 0.04 \\
GRUMA B & 0.00 & 0.09 & 0.07 & 0.90 & 0.02 \\
ARCA & 0.02 & 0.11 & 0.03 & 0.86 & 0.12 \\
CYDSASA A & -0.19 & 10.22 & 0.35 & 0.26 & 0.35 \\
SANLUIS CPO & -0.10 & 6.17 & 0.07 & 0.51 & 0.08 \\
BACHOCO UBL & 0.01 & 0.23 & 0.09 & 0.87 & 0.03 \\
ASUR B & 0.04 & 0.20 & 0.03 & 0.98 & 0.03 \\
VALLE V & -0.05 & 1.51 & 0.31 & 0.62 & 0.12 \\
\hline
\end{tabular}

Cuadro 3a. (continue).

\begin{tabular}{lcccc}
\hline Emisora & t-gama & $\sigma^{2}$ & betacapm & CoefDet \\
MASECA B & 4.06 & 9.86 & 0.40 & 0.06 \\
MOVILA B & 34.98 & 46.31 & 0.40 & 0.01 \\
USCOM B1 & 2.98 & 5.78 & 0.39 & 0.06 \\
IMSA UBC & 5.97 & 6.73 & 0.38 & 0.07 \\
GRUMA B & 2.08 & 6.17 & 0.36 & 0.08 \\
ARCA & 4.90 & 3.02 & 0.33 & 0.05 \\
CYDSASA A & 9.02 & 10.14 & 0.28 & 0.01 \\
SANLUIS CPO & 3.34 & 8.82 & 0.27 & 0.01 \\
BACHOCO UBL & 3.41 & 10.05 & 0.25 & 0.04 \\
ASUR B 0.04 & 25.02 & 8.96 & 0.21 & 0.01 \\
VALLE V & 6.14 & 5.92 & 0.15 & 0.01 \\
\hline
\end{tabular}

Enseguida presentamos algunas gráficas representativas del análisis: En primer lugar la curva de apalancamiento del IPC. En la figura 1, están dibujados los cambios en la volatilidad del mercado contra los choques de los rendimientos. La figura muestra que los choques impactan la volatilidad, es decir, la respuesta de la volatilidad a los choques está contenida en el impacto de la curva de noticias, la cual muestra asimetría. 
Gráfica 1. Curva del IPC.

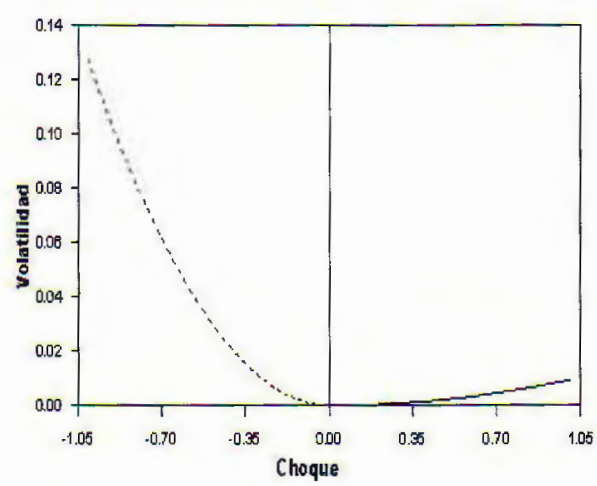

Después mostraremos las curvas de algunas firmas. Observamos que algunas de ellas tienen un alto y mediano apalancamiento por lo que la cruva de asimetría es pronunciada (alta) a causa de los choques del mercado. Por el contrario, cuando apalancamiento es bajo la asimetría es menos significativa y los choques tienen un fuerte impacto sobre la volatilidad

Gráfica 2: Curva de WALMEX_V.

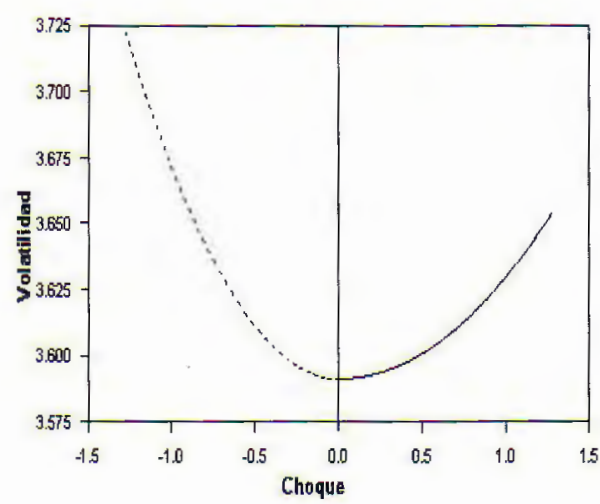


Gráfica 3. Curva de BIMBO_A.

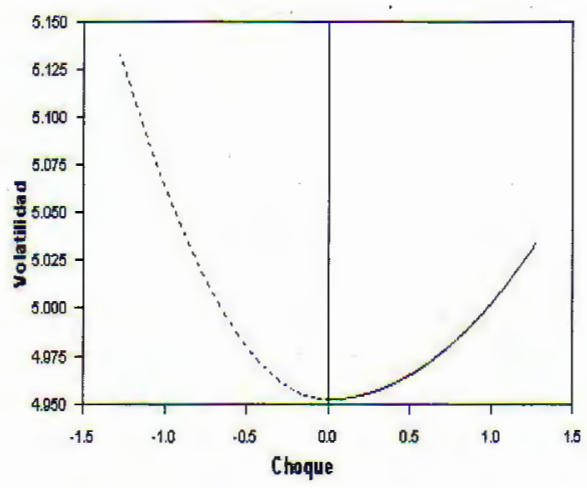

Gráfica 4. Curva de ICA.

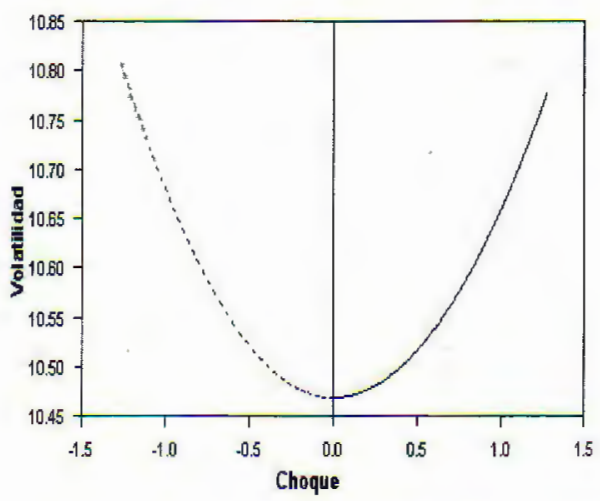

Gráfica 5. Curva de KIMBER_A.

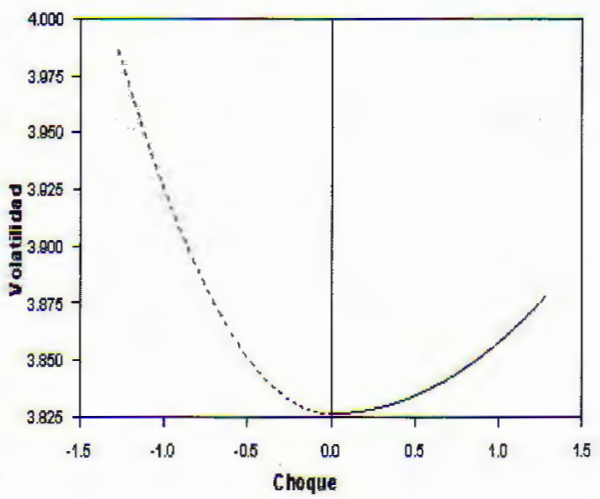


Gráfica 6. Curva de MASECABB.

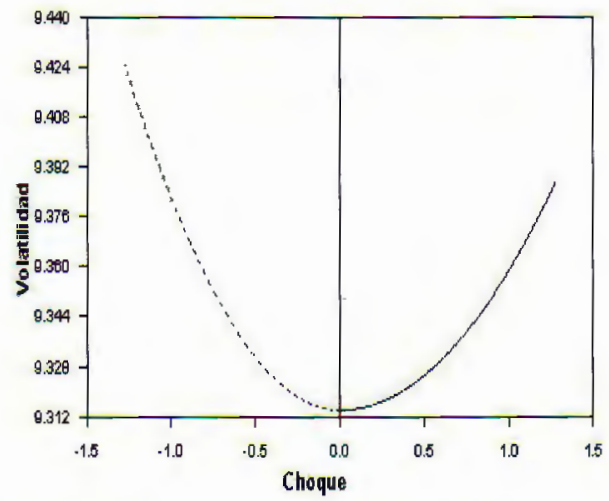

Gráfica 7. Curva de CEMEX_CPO.

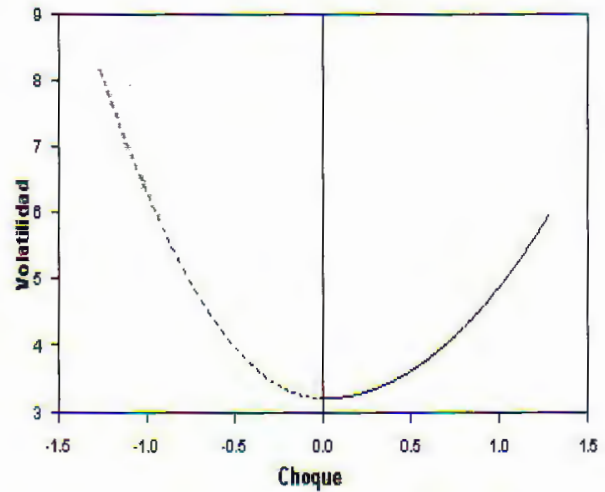

Gráfica 8. Curva de SORIANA_B.

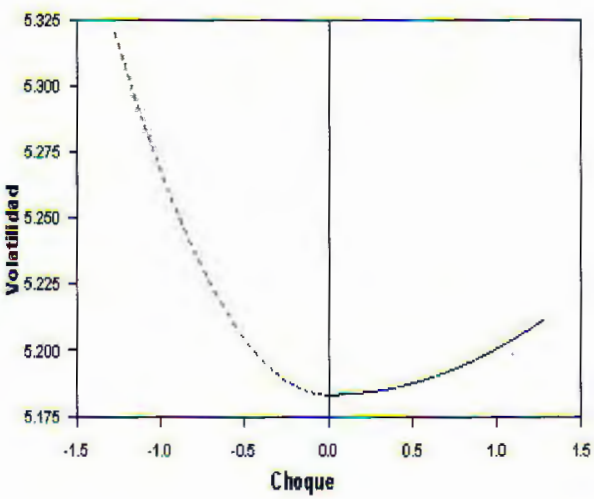


Gráfica 9. Curva de VITRO_ A.

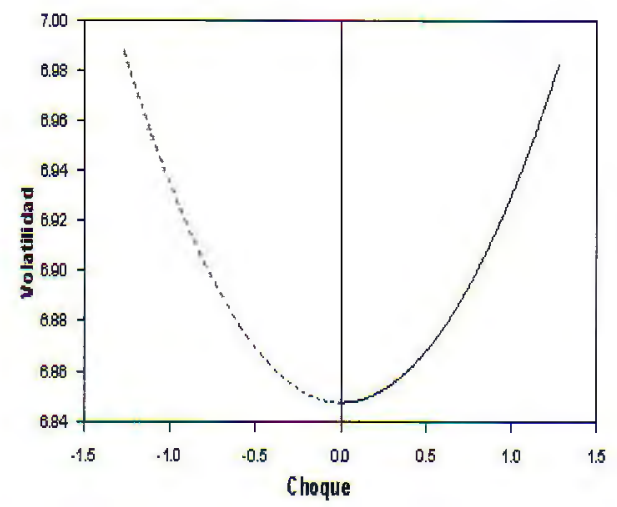

Gráfica 10. Curva de TELMEX_ L.

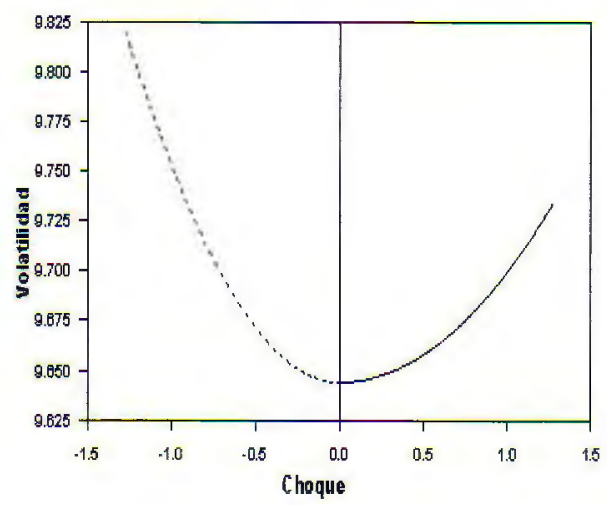

El cuadro 4 corresponde a los resultados del modelo exponencial de Nelson, tienen especial atención el parámetro gama y su t-gama. Cuando el valor de $t$ es significativo muestra que la curva de noticias si tiene un efcto asimétrico.

La columna PLUS muestra el impacto a que es forzada la volatilidad bajo un choque positivo, mientras que la columna MINUS muestra el impacto de las malas noticias, este efecto ocurre cuando una caída no esperada en el precio (malas noticias) incrementan la volatilidad mas fuerte que cuando presenta un incremento no esperado en el precio (buenas nuevas) de igual magnitud (Engel \& $\operatorname{Ng}(1993)$, pp. 1755). 
Cuadro 4. Los resultados del modelo Garch Exponencial (EGARCH) para las 62 emisoras.

\begin{tabular}{lccccc}
\hline Emisora & media & omega & alfa & beta & gama \\
ALFA & 0.104 & -0.061 & 0.117 & 0.988 & -0.036 \\
AMTEL A1 & 0.232 & -0.059 & 0.245 & 0.899 & -0.102 \\
AMX A & 0.130 & 0.099 & 0.092 & 0.974 & -0.094 \\
AMX L & -0.178 & 2.224 & 0.841 & -0.294 & -0.707 \\
ARA & 0.197 & 0.002 & 0.003 & 1.000 & -0.014 \\
ARCA & 0.038 & -0.091 & 0.177 & 0.950 & -0.097 \\
ASUR B & 0.229 & 0.093 & 0.075 & 0.966 & -0.054 \\
BACHOCO UBL & 0.037 & -0.004 & 0.144 & 0.947 & -0.027 \\
BIMBO A & 0.042 & -0.067 & 0.185 & 0.956 & -0.040 \\
C & -0.110 & 0.012 & 0.011 & 0.998 & -0.064 \\
CEL & 1.027 & 0.971 & 2.558 & 0.046 & -1.273 \\
CEMEX CPO & 0.086 & 0.102 & 0.546 & 0.739 & -0.199 \\
CIE B & 0.054 & -0.049 & 0.106 & 0.983 & -0.071 \\
CNCI B & -0.362 & 0.038 & 0.407 & 0.890 & -0.043 \\
COMERCI UBC & 0.048 & -0.096 & 0.198 & 0.969 & -0.035 \\
CONTAL & 0.036 & 3.640 & 0.056 & -0.978 & -0.027 \\
CYDSASA A & -0.348 & 0.657 & 0.217 & 0.718 & -0.113 \\
DESC B & 0.175 & 4.827 & 0.038 & -0.845 & -0.287 \\
ELEKTRA & -0.181 & 1.679 & 0.794 & 0.088 & -0.657 \\
FEMSA UBD & 0.180 & 3.358 & 0.086 & -0.971 & -0.002 \\
GCARSO A1 & 0.025 & 4.638 & 0.151 & -0.801 & -0.167 \\
GCC & 0.110 & -0.156 & 0.321 & 0.932 & -0.022 \\
GCORVI UBL & -0.057 & 0.024 & 0.074 & 0.965 & -0.002 \\
GEO B & 0.217 & -0.006 & 0.878 & 0.789 & -0.596 \\
GFINBUR O & 0.157 & 3.787 & 0.174 & -0.975 & -0.240 \\
GFNORTE O & 0.155 & -0.045 & 0.085 & 0.990 & -0.050 \\
GIGANTE & 0.000 & 0.163 & 0.052 & 0.937 & -0.039 \\
GISSA & -0.035 & -0.142 & 0.328 & 0.927 & -0.019 \\
GMEXICO B & -0.153 & 1.695 & 1.372 & -0.213 & -0.747 \\
GMODELO C & 0.055 & 2.896 & 0.148 & -0.861 & -0.143 \\
GRUMA B & -0.035 & -0.050 & 0.173 & 0.955 & -0.030 \\
GSANBOR B1 & 0.032 & -0.014 & 0.015 & 1.002 & -0.010 \\
HILASAL A & -0.104 & -0.028 & 0.240 & 0.949 & -0.002 \\
HOGAR B & -0.192 & 0.581 & 0.381 & 0.655 & -0.026 \\
\hline & & & & & \\
& & & &
\end{tabular}


Cuadro 4. (continuación).

\begin{tabular}{|c|c|c|c|c|c|c|}
\hline Emisora & t gamma & plus & minus & $\sigma^{2}$ & betacapm & Coefdet \\
\hline ALFA & -5.732 & 0.081 & 0.152 & 9.217 & 1.059 & 0.343 \\
\hline AMTEL A1 & -4.652 & 0.143 & 0.347 & 3.722 & 1.422 & 0.569 \\
\hline $\mathrm{AMX} A$ & -19.057 & 0.002 & 0.187 & 5.091 & 1.065 & 0.099 \\
\hline AMX L & -16.827 & 0.134 & 1.549 & 4.238 & 1.439 & 0.188 \\
\hline ARA & -23.758 & 0.010 & 0.017 & 6.132 & 0.684 & 0.106 \\
\hline ARCA & -6.103 & 0.080 & 0.274 & 2.985 & 0.333 & 0.054 \\
\hline ASUR B & -8.084 & 0.020 & 0.129 & 8.818 & 0.211 & 0.009 \\
\hline BACHOCOUBL & -4.529 & 0.118 & 0.171 & 9.933 & 0.253 & 0.040 \\
\hline BIMBO A & -4.339 & 0.144 & 0.225 & 5.411 & 0.620 & 0.211 \\
\hline $\mathrm{C}$ & -17.969 & 0.053 & 0.074 & 3.384 & 0.800 & 0.223 \\
\hline CEL & -16.984 & 1.285 & 3.831 & 1366.4 & -0.288 & 0.000 \\
\hline CEMEX CPO & -11.382 & 0.347 & 0.745 & 4.948 & 0.944 & 0.325 \\
\hline CIE B & -11.887 & 0.035 & 0.176 & 7.993 & 1.084 & 0.414 \\
\hline CNCI B & -4.552 & 0.363 & 0.450 & 21.720 & 0.940 & 0.089 \\
\hline COMERCIUBC & -4.440 & 0.163 & 0.233 & 6.484 & 0.758 & 0.271 \\
\hline CONTAL & -11.099 & 0.030 & 0.083 & 4.692 & 0.467 & 0.074 \\
\hline CYDSASA A & -11.918 & 0.104 & 0.330 & 10.380 & 0.280 & 0.012 \\
\hline DESC B & -92.917 & 0.249 & 0.326 & 8.373 & 0.811 & 0.077 \\
\hline ELEKTRA & -52.313 & 0.138 & 1.451 & 37.346 & 1.145 & 0.097 \\
\hline FEMSA UBD & -2.796 & 0.083 & 0.088 & 5.860 & 1.019 & 0.080 \\
\hline GCARSO A1 & -6.118 & 0.017 & 0.318 & 5.802 & 1.004 & 0.211 \\
\hline $\mathrm{GCC}$ & -1.978 & 0.299 & 0.343 & 3.405 & 0.287 & 0.053 \\
\hline GCORVI UBL & -0.449 & 0.072 & 0.076 & 3.854 & 0.388 & 0.062 \\
\hline GEO B & -29.852 & 0.281 & 1.474 & 6.586 & 0.855 & 0.043 \\
\hline GFINBUR O & -19.809 & 0.067 & 0.414 & 4.139 & 0.619 & 0.073 \\
\hline GFNORTE O & -6.866 & 0.034 & 0.135 & 6.875 & 0.985 & 0.331 \\
\hline GIGANTE & -7.184 & 0.012 & 0.091 & 53.459 & 0.282 & 0.015 \\
\hline GISSA & -1.684 & 0.309 & 0.347 & 0.977 & 0.232 & 0.028 \\
\hline GMEXICO B & -15.166 & 0.625 & 2.119 & 5.053 & 0.914 & 0.037 \\
\hline GMODELO C & -30.774 & 0.005 & 0.291 & 4.166 & 0.421 & 0.034 \\
\hline GRUMA B & -5.639 & 0.143 & 0.203 & 3.256 & 0.358 & 0.082 \\
\hline GSANBOR B1 & -3.593 & 0.006 & 0.025 & 5.869 & 0.624 & 0.177 \\
\hline HILASAL A & -0.389 & 0.238 & 0.242 & 7.186 & 0.541 & 0.078 \\
\hline HOGAR B & -1.462 & 0.354 & 0.407 & 9.946 & 0.500 & 0.055 \\
\hline
\end{tabular}


Cuadro 4. (continuación).

\begin{tabular}{lccccc}
\hline Emisora & media & omega & alfa & beta & gama \\
HOMEX & 0.150 & -0.101 & 0.537 & 0.785 & -0.062 \\
HYLSAMX B & 0.128 & -0.070 & 0.373 & 0.920 & -0.046 \\
HYLSAMX L & 0.102 & -0.191 & 0.459 & 0.934 & -0.151 \\
ICA & -0.103 & -0.023 & 0.111 & 0.980 & -0.007 \\
ICH B & 0.000 & 4.169 & 0.103 & -0.961 & -0.005 \\
IMSA UBC & 0.080 & -0.024 & 0.184 & 0.939 & -0.035 \\
KIMBER A & 0.027 & -0.087 & 0.160 & 0.977 & -0.060 \\
KOF L & 0.106 & -0.009 & 0.060 & 0.982 & -0.033 \\
MASECA B & 0.094 & -0.012 & 0.019 & 0.999 & -0.028 \\
MOVILA B & -0.160 & 2.287 & 1.111 & -0.010 & -0.566 \\
NAFTRAC 02 & 0.087 & -0.080 & 0.096 & 0.906 & -0.169 \\
PENOLES & -0.029 & 0.717 & 0.285 & 0.559 & -0.013 \\
SANLUIS CPO & -0.140 & 0.752 & 0.167 & 0.692 & -0.035 \\
SARE B & 0.085 & 0.212 & 0.271 & 0.574 & -0.016 \\
SAVIA A & 0.000 & -0.191 & 0.604 & 0.991 & -0.307 \\
SIMEC B & 0.000 & 0.686 & 0.215 & 0.842 & -0.197 \\
SORIANA B & 0.063 & -0.051 & 0.097 & 0.985 & -0.049 \\
TELECOM A1 & 0.124 & 0.002 & 0.006 & 1.001 & -0.009 \\
TELMEX A & 0.050 & 2.710 & 0.309 & -0.105 & -0.162 \\
TELMEX L & -0.077 & 3.066 & 0.267 & -0.292 & -0.015 \\
TLEVISA CPO & 0.067 & -0.057 & 0.093 & 0.993 & -0.037 \\
TS & 0.252 & 1.277 & 0.112 & 0.124 & -0.115 \\
TVAZTCA CPO & 0.026 & -0.069 & 0.143 & 0.984 & -0.033 \\
URBI & 0.219 & 0.009 & 0.119 & 0.905 & -0.006 \\
USCOM B1 & 0.000 & 0.213 & 0.553 & 0.414 & -0.098 \\
VALLE V & -0.144 & 0.153 & 0.436 & 0.811 & -0.035 \\
VITRO A & -0.096 & -0.046 & 0.177 & 0.961 & -0.017 \\
WALMEX V & 0.065 & -0.073 & 0.112 & 0.990 & -0.046 \\
\hline
\end{tabular}


Cuadro 4. (continuación).

\begin{tabular}{|c|c|c|c|c|c|c|}
\hline Emisora & t gamma & plus & minus & $\sigma^{2}$ & betacapm & Coefdet \\
\hline HOMEX & -1.428 & 0.475 & 0.599 & 5.545 & 0.721 & 0.106 \\
\hline HYLSAMX B & -3.539 & 0.327 & 0.418 & 15.477 & 0.692 & 0.058 \\
\hline HYLSAMX L & -3.975 & 0.307 & 0.610 & 5.017 & 0.903 & 0.136 \\
\hline $\mathrm{ICA}$ & -1.899 & 0.103 & 0.118 & 12.829 & 0.886 & 0.157 \\
\hline ICH B & -2.344 & 0.098 & 0.107 & 11.197 & 0.499 & 0.058 \\
\hline IMSA UBC & -5.754 & 0.149 & 0.218 & 6.695 & 0.376 & 0.072 \\
\hline KIMBER A & -8.226 & 0.099 & 0.220 & 4.069 & 0.703 & 0.315 \\
\hline $\mathrm{KOF} \mathrm{L}$ & -6.370 & 0.027 & 0.094 & 7.621 & 0.526 & 0.127 \\
\hline MASECA B & -11.011 & 0.010 & 0.047 & 9.729 & 0.397 & 0.063 \\
\hline MOVILA B & -24.840 & 0.544 & 1.677 & 46.285 & 0.397 & 0.008 \\
\hline NAFTRAC 02 & -5.907 & 0.073 & 0.265 & 1.006 & 0.937 & 0.913 \\
\hline PENOLES & -0.892 & 0.271 & 0.298 & 6.991 & 0.354 & 0.039 \\
\hline SANLUIS CPO & -2.766 & 0.132 & 0.203 & 8.885 & 0.266 & 0.012 \\
\hline SARE B & -0.355 & 0.256 & 0.287 & 3.786 & 0.699 & 0.172 \\
\hline SAVIA A & -122.24 & 0.297 & 0.911 & 27.787 & 0.405 & 0.009 \\
\hline SIMEC B & -49.793 & 0.017 & 0.412 & 275.25 & 0.462 & 0.008 \\
\hline SORIANA B & -6.483 & 0.048 & 0.146 & 5.462 & 0.913 & 0.420 \\
\hline TELECOM A1 & -3.540 & 0.003 & 0.014 & 6.266 & 1.074 & 0.179 \\
\hline TELMEX A & -7.893 & 0.147 & 0.471 & 4.820 & 0.718 & 0.122 \\
\hline TELMEX L & -0.656 & 0.253 & 0.282 & 4.156 & 0.992 & 0.249 \\
\hline TLEVISA CPO & -8.127 & 0.057 & 0.130 & 7.970 & 1.269 & 0.551 \\
\hline $\mathrm{TS}$ & -3.977 & 0.003 & 0.227 & 11.016 & 0.240 & 0.012 \\
\hline TVAZTCACPO & -5.949 & 0.110 & 0.175 & 11.670 & 1.254 & 0.397 \\
\hline URBI & -0.249 & 0.113 & 0.125 & 3.371 & 0.404 & 0.055 \\
\hline USCOM B1 & -2.420 & 0.456 & 0.651 & 5.862 & 0.385 & 0.056 \\
\hline VALLE V & -4.607 & 0.402 & 0.471 & 6.009 & 0.146 & 0.006 \\
\hline VITRO A & -2.109 & 0.159 & 0.194 & 7.532 & 0.562 & 0.109 \\
\hline WALMEX V & -4.858 & 0.066 & 0.159 & 3.815 & 1.092 & 0.524 \\
\hline
\end{tabular}

De esta tabla general se procede de igual forma primero se eligen las emisoras con un marcado efecto asimétrico y estas se ordenan por su valor betacamp obteniendo 51 emisoras. Las empresas que no se incluyen son: GCC, ICA, GISSA, HOGAR B,HOMEX, PENOLES, TELMEX L, GCORVI UBL, HILASAL A, SARE B, URBI. 
Cuadro 4. (continuación).

\begin{tabular}{lccccc}
\hline Emisora & media & omega & alfa & beta & gama \\
AMX L & -0.18 & 2.22 & 0.84 & -0.29 & -0.71 \\
AMTEL A1 & 0.23 & -0.06 & 0.25 & 0.90 & -0.10 \\
TLEVISA CPO & 0.07 & -0.06 & 0.09 & 0.99 & -0.04 \\
TVAZTCA CPO & 0.03 & -0.07 & 0.14 & 0.98 & -0.03 \\
ELEKTRA & -0.18 & 1.68 & 0.79 & 0.09 & -0.66 \\
WALMEX & 0.06 & -0.07 & 0.11 & 0.99 & -0.05 \\
CIE B & 0.05 & -0.05 & 0.11 & 0.98 & -0.07 \\
TELECOM A1 & 0.12 & 0.00 & 0.01 & 1.00 & -0.01 \\
AMX A & 0.13 & 0.10 & 0.09 & 0.97 & -0.09 \\
ALFA & 0.10 & -0.06 & 0.12 & 0.99 & -0.04 \\
FEMSA UBD & 0.18 & 3.36 & 0.09 & -0.97 & 0.00 \\
GCARSO A1 & 0.02 & 4.64 & 0.15 & -0.80 & -0.17 \\
GFNORTE O & 0.15 & -0.05 & 0.08 & 0.99 & -0.05 \\
CEMEX CPO & 0.09 & 0.10 & 0.55 & 0.74 & -0.20 \\
CNCI B & -0.36 & 0.04 & 0.41 & 0.89 & -0.04 \\
NAFTRAC O2 & 0.09 & -0.08 & 0.10 & 0.91 & -0.17 \\
GMEXICO B & -0.15 & 1.69 & 1.37 & -0.21 & -0.75 \\
SORIANA B & 0.06 & -0.05 & 0.10 & 0.99 & -0.05 \\
HYLSAMX 1 & 0.10 & -0.19 & 0.46 & 0.93 & -0.152 \\
GEO B & 0.22 & -0.01 & 0.88 & 0.79 & -0.60 \\
DESC B & 0.17 & 4.83 & 0.04 & -0.85 & -0.29 \\
C & 0.01 & 0.01 & 0.01 & 1.00 & -0.06 \\
COMERCI UBC & 0.05 & -0.10 & 0.20 & 0.97 & -0.03 \\
TELMEX A & 0.05 & 2.71 & 0.31 & -0.11 & -0.16 \\
KIMBER A & 0.03 & -0.09 & 0.16 & 0.98 & -0.06 \\
HYLSAMX B & 0.13 & -0.07 & 0.37 & 0.92 & -0.05 \\
ARA & 0.20 & 0.00 & 0.00 & 1.00 & -0.01 \\
GSANBORS B1 & 0.03 & -0.01 & 0.02 & 1.00 & -0.01 \\
BIMBO A & 0.04 & -0.07 & 0.18 & 0.96 & -0.04 \\
GFINBUR O & 0.16 & 3.79 & 0.17 & -0.98 & -0.24 \\
VITRO A & -0.10 & -0.05 & 0.18 & 0.96 & -0.02 \\
KOF L & 0.11 & -0.01 & 0.06 & 0.98 & -0.03 \\
ICH B & 0.00 & 4.17 & 0.10 & -0.96 & 0.00 \\
CONTAL & -0.04 & 3.64 & 0.06 & -0.98 & -0.03 \\
\hline & & & & & \\
& & & &
\end{tabular}


Cuadro 4. (continuación).

\begin{tabular}{lcccccc}
\hline Emisora & $\mathrm{t}$ gamma & plus & minus & $\sigma^{2}$ & betacapm & Coefdet \\
AMX L & -16.83 & 0.13 & 1.55 & 4.24 & 1.44 & 0.19 \\
AMTEL A1 & -4.65 & 0.14 & 0.35 & 3.72 & 1.42 & 0.57 \\
TLEVISA CPO & -8.13 & 0.06 & 0.13 & 7.97 & 1.27 & 0.55 \\
TVAZTCA CPO & -5.95 & 0.11 & 0.18 & 11.67 & 1.25 & 0.40 \\
ELEKTRA & -52.31 & 0.14 & 1.45 & 37.35 & 1.14 & 0.10 \\
WALMEX & -4.86 & 0.07 & 0.16 & 3.81 & 1.09 & 0.52 \\
CIE B & -11.89 & 0.04 & 0.18 & 7.99 & 1.08 & 0.41 \\
TELECOM A1 & -3.54 & 0.00 & 0.01 & 6.27 & 1.07 & 0.18 \\
AMX A & -19.06 & 0.00 & 0.19 & 5.09 & 1.06 & 0.10 \\
ALFA & -5.73 & 0.08 & 0.15 & 9.22 & 1.06 & 0.34 \\
FEMSA UBD & -2.80 & 0.08 & 0.09 & 5.86 & 1.02 & 0.08 \\
GCARSO A1 & -6.12 & 0.02 & 0.32 & 5.80 & 1.00 & 0.21 \\
GFNORTE O & -6.87 & 0.03 & 0.14 & 6.88 & 0.99 & 0.33 \\
CEMEX CPO & -11.38 & 0.35 & 0.75 & 4.95 & 0.94 & 0.32 \\
CNCI B & -4.55 & 0.36 & 0.45 & 21.72 & 0.94 & 0.09 \\
NAFTRAC O2 & -5.91 & 0.07 & 0.27 & 1.01 & 0.94 & 0.91 \\
GMEXICO B & -15.17 & 0.62 & 2.12 & 5.05 & 0.91 & 0.04 \\
SORIANA B & -6.48 & 0.05 & 0.15 & 5.46 & 0.91 & 0.42 \\
HYLSAMX 1 & -3.97 & 0.31 & 0.61 & 5.02 & 0.90 & 0.14 \\
GEO B & -29.85 & 0.28 & 1.47 & 6.59 & 0.86 & 0.04 \\
DESC B & -92.92 & 0.25 & 0.33 & 8.37 & 0.81 & 0.08 \\
C & -17.97 & 0.05 & 0.07 & 3.38 & 0.80 & 0.22 \\
COMERCI UBC & -4.44 & 0.16 & 0.23 & 6.48 & 0.76 & 0.27 \\
TELMEX A & -7.89 & 0.15 & 0.47 & 4.82 & 0.72 & 0.12 \\
KIMBER A & -8.23 & 0.10 & 0.22 & 4.07 & 0.70 & 0.31 \\
HYLSAMX B & -3.54 & 0.33 & 0.42 & 15.48 & 0.69 & 0.06 \\
ARA & -23.76 & 0.01 & 0.02 & 6.13 & 0.68 & 0.11 \\
GSANBORS B1 & -3.59 & 0.01 & 0.02 & 5.87 & 0.62 & 0.18 \\
BIMBO A & -4.34 & 0.14 & 0.23 & 5.41 & 0.62 & 0.21 \\
GFINBUR O & -19.81 & 0.07 & 0.41 & 4.14 & 0.62 & 0.07 \\
VITRO A & -2.11 & 0.16 & 0.19 & 7.53 & 0.56 & 0.11 \\
KOF L & -6.37 & 0.03 & 0.09 & 7.62 & 0.53 & 0.13 \\
ICH B & -2.34 & 0.10 & 0.11 & 11.20 & 0.50 & 0.06 \\
CONTAL & -11.10 & 0.03 & 0.08 & 4.69 & 0.47 & 0.07 \\
\hline & & & & & & \\
& & & & & & \\
& & & & &
\end{tabular}


Cuadro 4. (continuación).

\begin{tabular}{lccccc}
\hline Emisora & media & omega & alfa & beta & gama \\
SIMEC B & 0.00 & 0.69 & 0.21 & 0.84 & -0.20 \\
GMODELO C & 0.06 & 2.90 & 0.15 & -0.86 & -0.14 \\
SAVIA A & 0.00 & -0.19 & 0.60 & 0.99 & -0.31 \\
MASECA B & 0.09 & -0.01 & 0.02 & 1.00 & -0.03 \\
MOVILA A & -0.16 & 2.29 & 1.11 & -0.01 & -0.57 \\
USCOM B1 & 0.00 & 0.21 & 0.55 & 0.41 & -0.10 \\
IMSA UBC & 0.08 & -0.02 & 0.18 & 0.94 & -0.03 \\
GRUMA B & -0.04 & -0.05 & 0.17 & 0.95 & -0.03 \\
ARCA & 0.04 & -0.09 & 0.18 & 0.95 & -0.10 \\
GIGANTE & 0.00 & 0.16 & 0.05 & 0.94 & -0.04 \\
CYDSASA A & -0.35 & 0.66 & 0.22 & 0.72 & -0.11 \\
SAN LUIS CPO & -0.14 & 0.75 & 0.17 & 0.69 & -0.04 \\
BACHOCO UBL & 0.04 & 0.00 & 0.14 & 0.95 & -0.03 \\
TS & 0.25 & 1.28 & 0.11 & 0.12 & -0.11 \\
ASUR B & 0.23 & 0.09 & 0.07 & 0.97 & -0.05 \\
VALLE V & -0.14 & 0.15 & 0.44 & 0.81 & -0.03 \\
\hline
\end{tabular}

Cuadro 4. (continuación).

\begin{tabular}{lcccccc}
\hline Emisora & t gamma & plus & minus & $\sigma^{2}$ & betacapm & Coefdet \\
SIMEC B & -49.97 & 0.02 & 0.41 & 275.25 & 0.46 & 0.01 \\
GMODELO C & -30.77 & 0.01 & 0.29 & 4.17 & 0.42 & 0.03 \\
SAVIA A & -122.24 & 0.30 & 0.91 & 27.79 & 0.40 & 0.01 \\
MASECA B & -11.01 & 0.01 & 0.05 & 9.73 & 0.40 & 0.06 \\
MOVILA A & -24.84 & 0.54 & 1.68 & 46.29 & 0.40 & 0.01 \\
USCOM B1 & 2.42 & 0.46 & 0.65 & 5.86 & 0.39 & 0.06 \\
IMSA UBC & -5.75 & 0.15 & 0.22 & 6.70 & 0.38 & 0.07 \\
GRUMA B & 5.64 & 0.14 & 0.20 & 3.26 & 0.36 & 0.08 \\
ARCA & -6.10 & 0.08 & 0.27 & 2.98 & 0.33 & 0.05 \\
GIGANTE & -7.18 & 0.01 & 0.09 & 53.46 & 0.28 & 0.02 \\
CYDSASA A & -11.92 & 0.10 & 0.33 & 10.38 & 0.28 & 0.01 \\
SAN LUIS CPO & -2.77 & 0.13 & 0.20 & 8.88 & 0.27 & 0.01 \\
BACHOCO UBL & -4.53 & 0.12 & 0.17 & 9.93 & 0.25 & 0.04 \\
TS & -3.98 & 0.00 & 0.23 & 11.02 & 0.24 & 0.01 \\
ASUR B & -8.08 & 0.02 & 0.13 & 8.82 & 0.21 & 0.01 \\
VALLE V & -4.61 & 0.40 & 0.47 & 6.01 & 0.15 & 0.01 \\
\hline
\end{tabular}

Como observamos los resultados obtenidos en ambos modelos son similares y nuevamente las gráficas resultantes del modelo EGARCH son parecidas a las generadas por el modelo asimétrico. 
Gráfica 11. Curva de GSANBOR_B1.

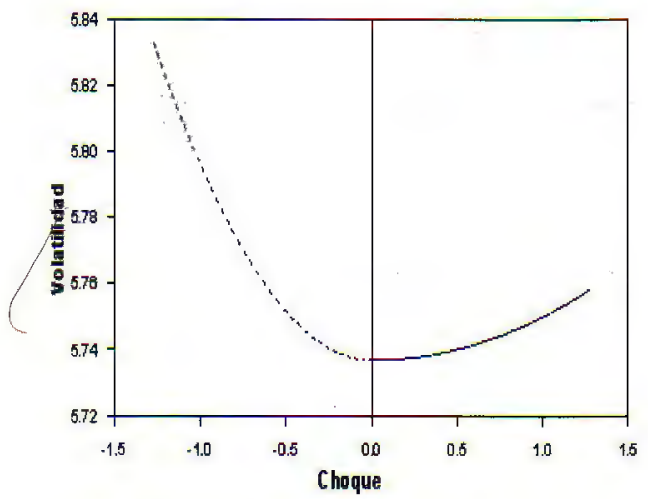

Gráfica 12. Curva de ALFA.

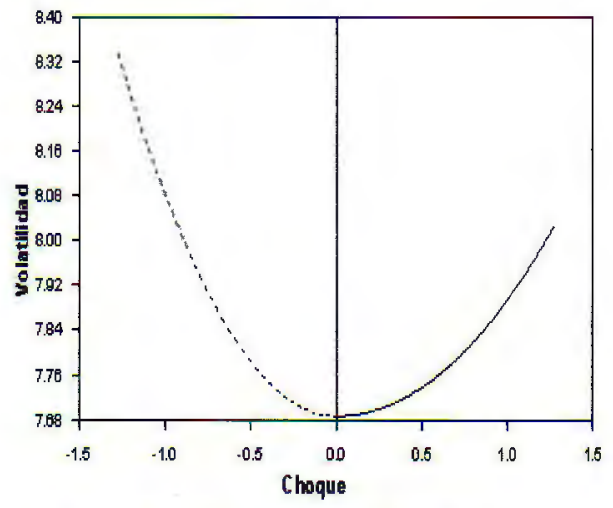

Gráfica 13. Curva de GIGANTE.

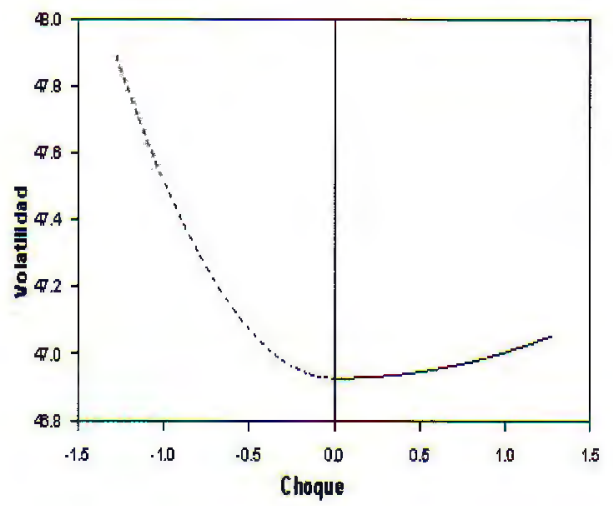


Gráfica 14. Curva de ARCA.

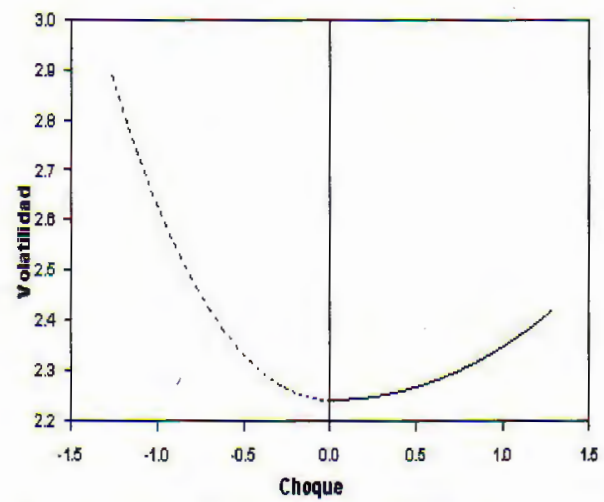

Gráfica 15. Curva de BIMBO_A.

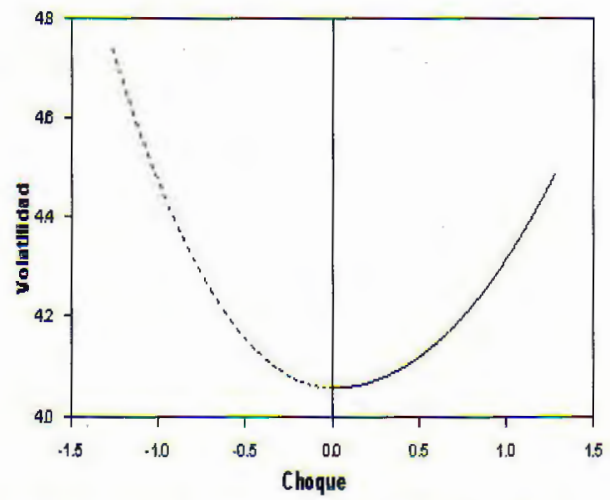

Gráfica 16. Curva de CEMEX_CPO.

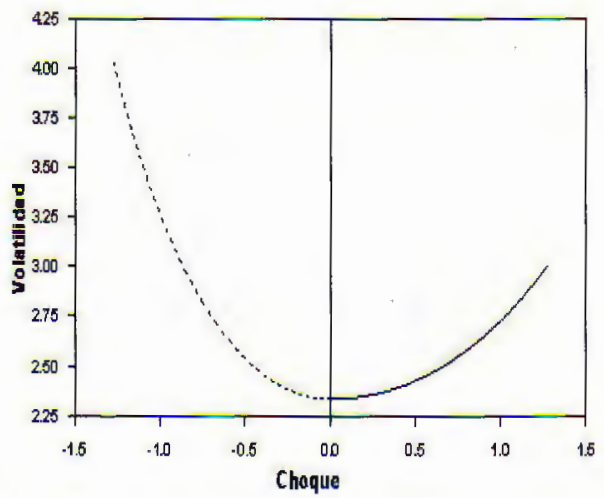

Las curvas de Gigante y Gsanbor son menos pronunciadas que con el modelo asimétrico. 
Gráfica 17. Curva de ICA.

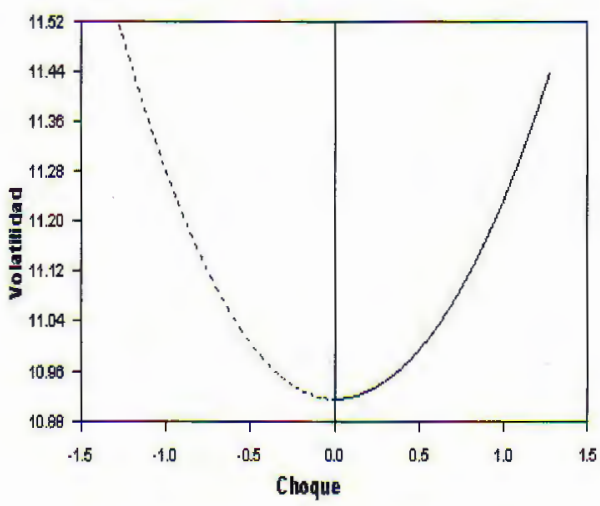

Gráfica 18. Curva de KIMBER_A.

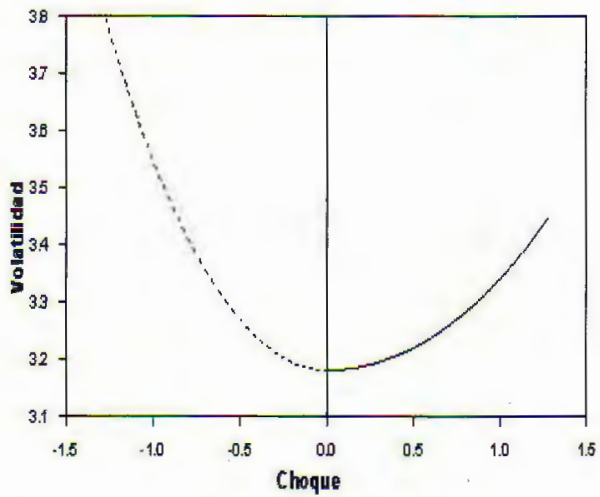

Gráfica 19. Curva de SORIANA_B.

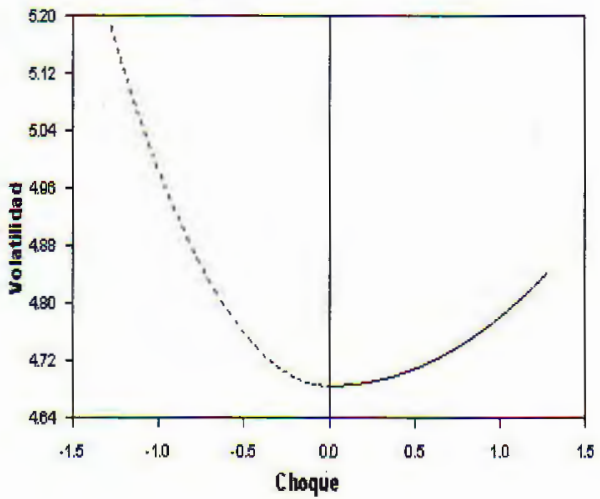


Gráfica 20. Curva de MASECA_B.

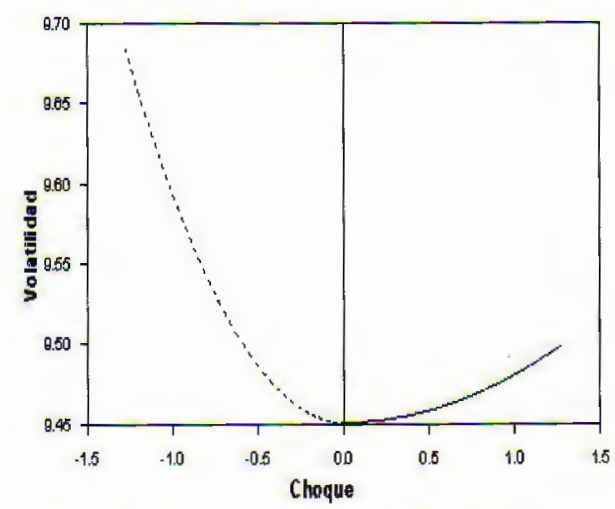

Gráfica 21. Curva de NAFTRAC_O2.

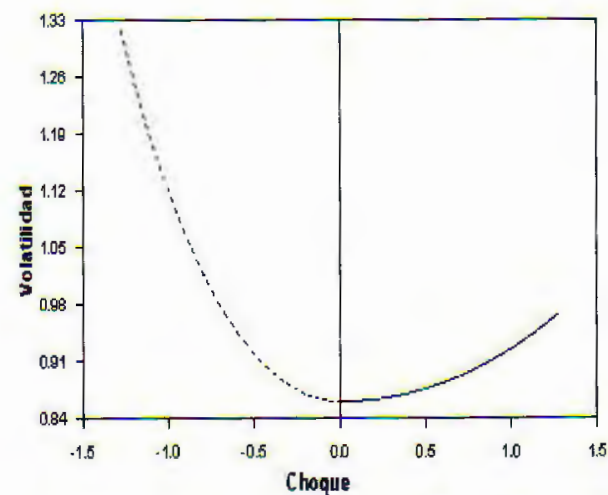

DE esta sección llaman la atención las curvas de SIMEC y TS.

Gráfica 22. Curva de SIMEC_B.

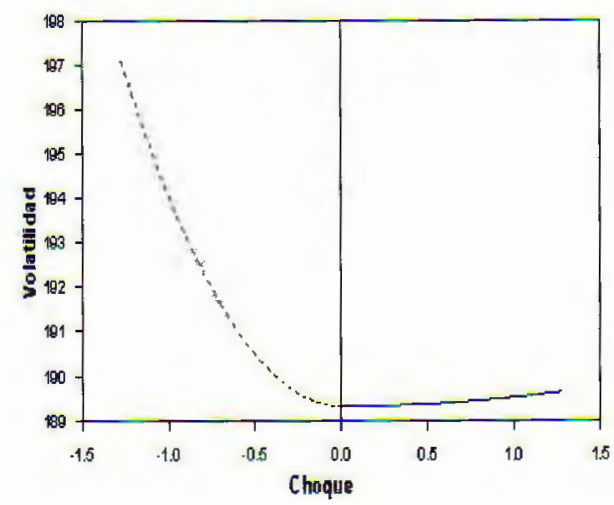


Gráfica 23. Curva de WALMEX_V.

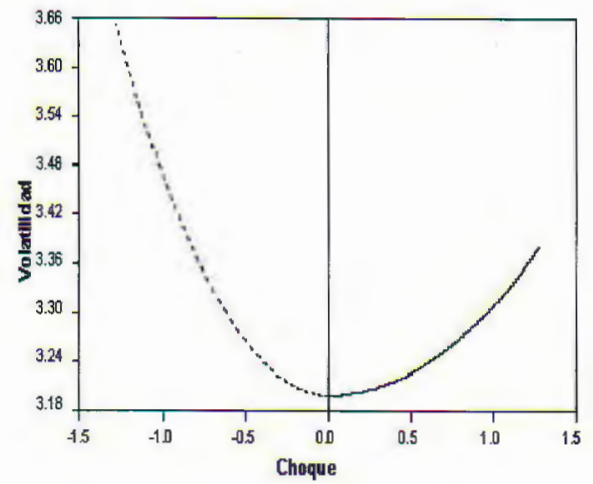

Gráfica 24. Curva de TELMEX L.

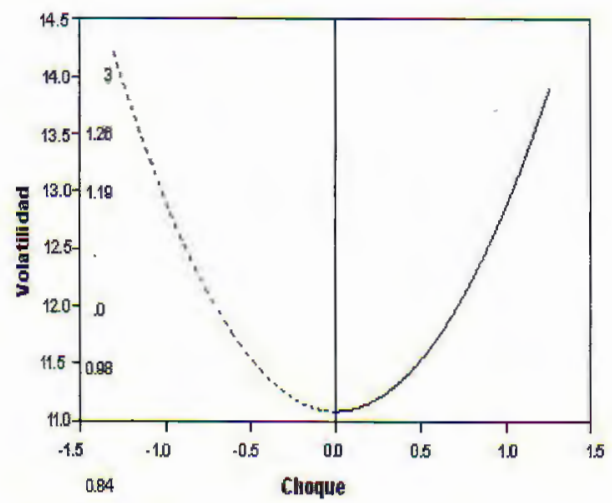

Gráfica 25. Curva de VITRO_A.

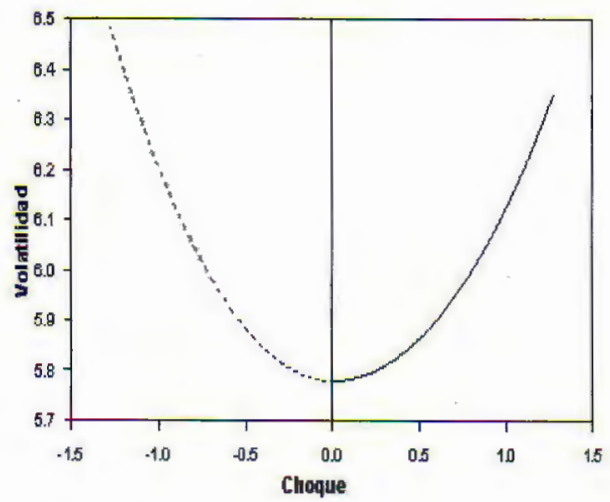


Gráfica 26. Curva de TS.

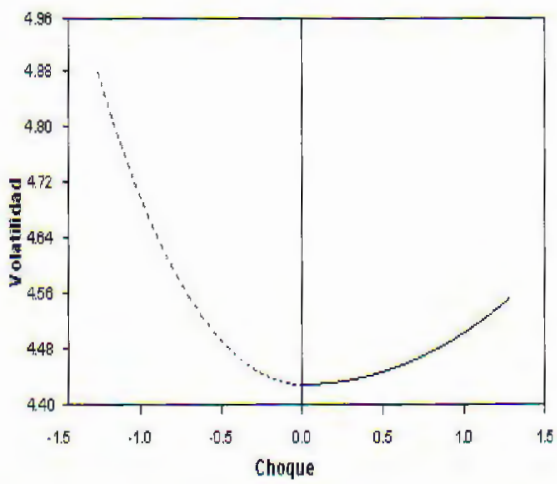

De acuerdo con los resultados obtenidos podemos concluir que es importante para los inversionistas modelar el comportamiento del mercado y la dinámica de las acciones de la bolsa en el largo plazo, para conocer los rendimientos esperados y a partir de esta información generar estrategias de administración de riesgo. Las curvas de apalancamiento nos permiten revisar los choques del mercado y de la acción provocados por la información asimétrica y los consecuentes cambios en la volatilidad.

Así, una estrategia de administración de riesgo podría ser la posibilidad de mantener una posición abierta en el mercado de opciones; una opción put generaría la posibilidad de vender el activo (la acción) cuando se esperara una caída en el precio de la acción y esto sucedería teóricamente cuando las malas noticias marcaran un choque negativo mas allá del nivel aceptado por el administrador de riesgos entonces la curva de apalancamiento de la emisora se pronunciaría mostrando el impacto de la volatilidad sobre los rendimientos de la acción. Al ejercer la opción eliminaríamos la posibilidad de perdidas.

En el diseño de una estrategia de administración de riesgo de esta naturaleza debemos considerar la volatilidad condicional futura y la covarianza entre los diferentes activos relacionados y es aquí donde se ligan el efecto apalancamiento y la teoría de opciones.

Estimar la volatilidad basados en la volatilidad implícita del precio de las opciones, bajo el supuesto de una volatilidad constante, y arbitraje financiero está documentado en Black y Scholes (1973) y Merton (1973) como la teoría del precio de las opciones. Intuitivamente, si los rendimientos de una opción pueden ser replicados por una cartera de otros activos, entonces el valor de la opción podría ser igual al valor de esa cartera o podría dar oportunidades de arbitraje. Por otro lado, la revisión de un proceso de volatilidad estocástica es analizado en Hull y White (1987), Engle y Mustafa (1992).

Por último, retomando lo señalado en la sección 2 de este trabajo: "La volatilidad de las acciones se incrementa cuando los rendimientos son negativos y desciende cuando los rendimientos son positivos", Black (1976). Este efecto es 
particularmente importante para el mercado de opciones ya que en realidad el precio de la opción refleja el hecho de que una correlación negativa rendimientovolatilidad induce a una distribución de rendimientos negativa asimétrica en el largo plazo.

\section{Conclusiones}

Observamos una clara persistencia en el tiempo de los choques de la varianza (una característica importante del mercado accionario) en los rendimientos del IPC así como de las acciones estudiadas. La volatilidad del mercado y de las acciones individuales se incrementa con las malas noticias y decrece con las buenas noticias. Es claro que las betas cambian asimétricamente en respuesta a las noticias, tal como se muestra en la sección 4. Es común observar que en el mercado mexicano crece el nerviosismo cuando hay caídas en los precios.

Debido al grado de significancia que muestran los parámetros de los modelos GARCH asimétrico y EGARCH exponencial utilizados en este estudio, podemos concluir que resultan una buena aproximación para estimar la asimetría de la volatilidad.

Por último, una sugerencia para el mercado bursátil mexicano es que sería interesante incluir en sus publicaciones además de las betas (que ya lo hace), la curva de noticias de cada emisora, estas curvas aceptadas mundialmente ayudan muchísimo al análisis financiero muy útil para comprender la dinámica del mercado. Por otro lado, es necesario ampliar el tema acerca de la consideración de incluir opciones put como estrategia de administración de riesgo en el efecto apalancamiento ya que ayuda en el campo profesional a tomar mejores decisiones.

\section{Bibliografía}

Akerlof, G. (1970). The Market for "lemons": Quality Uncertainty and the Market Mechanism. Quartely Journal Economics, August.

Black, F. (1976). Studies of Stock Market Volatility Changes, Proceedings of American Statistical Association, Business and Economic Statistics Section, 177-181.

Black, F. and M. Scholes (1973). The Pricing of Options and Corporate Liabilities. Journal of Political Economy, 81(3), 637-654.

Bollerslev, T., R. F. Engle, and J.M. Wooldridge (1988). A Capital Asset Pricing Model with Time Varynig Covariances. Journal of Political Economy, 96, 116-131.

Cheung, Y. and L. Ng (1992). Stock Price Dynamics and Firm Size: An Empirical Investigation. Journal of Finance, 47, 1985-1997.

Christie, A. (1982). The Stochastic Behavior of Common Stock Variances: Value, Leverage and Interest Rate Effects. Journal of Financial Economics, 10, 407-432.

Engle, R. and Ch. Mustafa (1992). Implied ARCH models from options prices. Journal of Econometrics.

Engle, R. and V. Ng (1993). Measuring and Testing the Impact of News on Volatility. Journal of Finance, $48,1749-1179$.

Engle, R., M. Lilien, and P. Robins (1987). Estimating Time Varying Risk Premia in the Term Structure: The ARCH-M Model.Econometrica, 55, 391-407.

Frech, K.R., G.W., Schwert and R.F. Stambaugh (1987). Expected Stock Returns and Volatility. Journal of Political Economy, 99, 385-415.

Glosten, L. R., R. Jagannathan, and D. E. Runkle (1993). On the Relation Between the Expect Value and the Volatility of the Nominal Excess Return on Stocks. Journal of Finance, 48, 1779-1801. 
Guo, H. (2002). Understanding the Risk-Return Tradeoff in the Stock Market. Working Paper No. 2002-001A, Federal Reserve Bank of St. Louis.

Hull, J. and A. White (1987). The Pricing of Options on Assets with Stochastic Volatilities. Journal of Finance, 42, 281-300.

Merton, R. (1973). An Intertemporal Capital Asset Ppricing Model Econometrica, 41(5), 867-887.

Merton, R. C. (1980). On Estimating the Expected Return on the Market: An Exploratory Investigation. Journal of Financial Economics, 8(4), 323-361.

$\mathrm{Ng}, \mathrm{L}$. (1991). Test of the CAPM with time varing covariances: A multivariate GARCH approach. Journal of Finance, 46, 1507-1523.

Pindyck, R. S. (1988). Risk Aversion and Determinants of Stock Market Behavior, Review of Economics and Statistics, 70(2), 183-190.

Nelson, D. B. (1989). Modeling Stock market volatility changes. Proceedings of the American Statistical Association. Business and Economics Statistics Section, 93-98.

Nelson, D. B. (1991). Conditional Heteroskedasticity in Asset Returns: a New Approach. Econometrica, 59, 2, 347-370.

Perrotini, A. (). La economía de la información asimétrica. Facultad de Economía de la BUAP, VII, 19, 59-67.

Proterba, T. M. and L.H. Summers (1986). The Persistence of Volatility and Stock Market Fluctuations.American Economic Review, 76, 1142-1151.

Turner, Ch., R. Startz, and Ch. Nelson (1989). A Markov Model of Heteroskedasticity, Risk and Learning in the Stock Market. Journal of Financial Economics, 25(1), 3-22.

Zakoian, J. (1990). Threshold Heteroskedastic Model, Unpublished manuscript (INSEE, París). 